\title{
CSF Biomarkers for Alzheimer Disease Diagnosis: Recent and Future Perspectives
}

\author{
Sebastiaan Engelborghs $\mathbf{s}^{1,2 *}$ \\ ${ }^{1}$ Reference Center for Biological Markers of Dementia (BIODEM), University of Antwerp, Belgium \\ ${ }^{2}$ Memory Clinic, Hospital Network Antwerp (ZNA), Antwerp, Belgium
}

Until recently, Alzheimer's disease (AD) was diagnosed clinically by excluding other conditions and pathologies, at best resulting in a diagnosis of probable $\mathrm{AD}$ [1]. Compared to autopsy-confirmed diagnoses, only $68 \%$ of the dementia patients were correctly diagnosed using these clinical diagnostic criteria for $\operatorname{AD}[2,3]$. Moreover, a clinical diagnosis of probable $\mathrm{AD}$ was confined to the dementia stage of the disease, following several years of progressive cognitive deterioration and behavioral changes. The neuropathological brain lesions that consist of senile plaques and neurofibrillary tangles, start developing many years before the first symptoms of the disease appear.

Due to recent insights, $\mathrm{AD}$ is no longer considered a clinical dementia syndrome but a progressive pathogenic process that can accurately be identified, also in its earliest stages. Therefore, revised diagnostic criteria for AD were published in 2011 [4-7]. These criteria better reflect the full continuum of $\mathrm{AD}$, from the preclinical over the predementia (mild cognitive impairment, MCI) to the full-blown dementia stages. These revised criteria include cerebrospinal fluid (CSF), imaging and genetic markers that provide biological evidence for $\mathrm{AD}$.

As the brain is in direct contact with the CSF and the flow of proteins to and from the CSF is restricted by the blood-CSF barrier, biochemical changes that reflect pathophysiological processes in the brain are reflected in CSF. The pathological hallmarks of AD are senile plaques and neurofibrillary tangles, which are made up of $\beta$-amyloid protein $(\mathrm{A} \beta)$ and hyperphosphorylated protein tau (P-tau), respectively. $\mathrm{A} \beta$, total tau (T-tau) and $\mathrm{P}$-tau proteins can reliably be measured in CSF by means of single-analyte ELISAs or a multianalyte test based on xMAP technology [8]. However, variations in biomarker measurements have been reported, both between and within laboratories [9]. Even when using the same assay, considerable variability in concentrations of $\mathrm{AD}$ biomarkers has been found between different centers leading to high variability and different cutoff values. The variations in concentration and diagnostic performance of biomarkers across studies could be the result of several preanalytical and analytical factors [10]. Assay-related factors (within and between plate and lot) arise from manufacturing variations in the source material for components and reagents in the analytical kits and random variability of the production process. All these items need careful standardization. As such variations jeopardize the introduction of CSF biomarkers in clinical routine and clinical trials, a number of national and international standardization and harmonization initiatives have been initiated which already resulted in a harmonized pre-analytical protocol for CSF biomarkers [11].

Besides the need for improved standardization and harmonization, what is the actual position of the CSF biomarkers A $\beta, T$-tau, and P-tau for diagnosing $\mathrm{AD}$ ? In which (differential) diagnostic situations can they be applied? The clinical and diagnostic usefulness and validity of these CSF biomarkers is supported by numerous studies in large and well-defined patient cohorts. In comparison to cognitively healthy elderly (including patients with psychiatric disorders like depression), the CSF biomarker profile in $\mathrm{AD}$ patients consists of decreased $\mathrm{A} \beta_{1-42}$ and increased T-tau and P-tau ${ }_{181 \mathrm{P}}$ levels. In order to improve the diagnostic accuracy, a combination of CSF biomarkers has been proposed. Some studies have addressed this by calculating ratios or developing biomarker models, which has led to a considerable improvement in diagnostic accuracy. A model based on $\mathrm{A} \beta_{1-42}$ and T-tau levels was developed that could accurately discriminate $\mathrm{AD}$ from controls by means of a discrimination line. This model has been validated in autopsy-confirmed dementia patients, resulting in sensitivity and specificity figures of $100 \%$ and $91 \%$ [3].

Can CSF biomarkers be applied in patients with mixed neurodegenerative and vascular dementias? As age is the most important risk factor for both neurodegenerative brain disorders and cerebrovascular disease (CVD), many dementia patients have CVD on brain magnetic resonance imaging (MRI) as was confirmed in neuropathological studies. The extent to which these lesions contribute to the clinical symptoms is unclear. Vascular brain injury may in itself cause dementia, but can also trigger amyloid deposition. In case of doubt between vascular dementia $(\mathrm{VaD})$ or mixed $\mathrm{AD}-\mathrm{VaD}$ pathology in dementia patients, the determination of CSF A $\beta_{1-42}$, T-tau and $\mathrm{P}-\mathrm{tau}_{181 \mathrm{P}}$ levels is of help to confirm or exclude the $\mathrm{AD}$ component in the pathophysiology of the dementia syndrome [12].

Can CSF biomarkers be used for diagnosing $\mathrm{AD}$ in the predementia stages of the disease? In MCI patients, concentrations of $\mathrm{A} \beta_{1-42}$, T-tau and $\mathrm{P}-\mathrm{tau}_{181 \mathrm{P}}$ in CSF are strongly associated with the future development of dementia, which was demonstrated in a large prospective study and in several multi-centre studies [13,14]. Also, a CSF AD profile is much more common in patients with MCI and subjective cognitive impairment [15]. In MCI patients with typical clinical symptoms for $\mathrm{AD}$ (progressive episodic memory loss) and biomarker evidence of $\mathrm{AD}$, the diagnosis should be 'MCI due to $\mathrm{AD}$ ', referring to the predementia stage of $\mathrm{AD}$ [4]. Since the pathological features of $\mathrm{AD}$ are present in nondemented elderly, indicative of preclinical $\mathrm{AD}$, one can assume that CSF biomarkers levels are also altered before the onset of symptoms. Furthermore, $36 \%$ of cognitively healthy elderly showed an AD-like biomarker profile and the T-tau/ $\mathrm{A} \beta_{1-42}$ and $\mathrm{P}-\mathrm{tau}_{18 \mathrm{P}} / \mathrm{A} \beta_{1-42}$ ratios in CSF are promising preclinical biomarkers that might predict cognitive decline in cognitively normal elderly [16,17]. However, studies with longer follow-up periods

*Corresponding author: Sebastiaan Engelborghs, MD, PhD, Reference Center for Biological Markers of Dementia (BIODEM), University of Antwerp, Antwerpen, Belgium, E-mail: sebastiaan.engelborghs@ua.ac.be

Received January 11, 2013; Accepted January 12, 2013; Published January 15, 2013

Citation: Engelborghs S (2013) CSF Biomarkers for Alzheimer Disease Diagnosis: Recent and Future Perspectives. J Neurol Disord 1:e102. doi:10.4172/2329 $6895.1000 \mathrm{e} 102$

Copyright: @ 2013 Engelborghs S. This is an open-access article distributed under the terms of the Creative Commons Attribution License, which permits unrestricted use, distribution, and reproduction in any medium, provided the original author and source are credited. 
Citation: Engelborghs S (2013) CSF Biomarkers for Alzheimer Disease Diagnosis: Recent and Future Perspectives. J Neurol Disord 1:e102. doi:10.4172/2329-6895.1000e102

are necessary to accurately describe the predictive value of CSF biomarkers, especially in healthy elderly.

How well do these CSF biomarkers perform for an $\mathrm{AD}$ versus non-AD dementia differential diagnosis? In comparison to non- $\mathrm{AD}$ dementias, differences in CSF A $\beta_{1-42}$, T-tau and P-tau ${ }_{181 \mathrm{P}}$ biomarker concentrations are much less pronounced as compared to cognitively healthy elderly. In general, both $\mathrm{A} \beta_{1-42}$ and T-tau in patients with non$\mathrm{AD}$ dementias are intermediate between the concentrations that are found in controls and patients with $\mathrm{AD}$, indicative of a large overlap in concentrations found in $\mathrm{AD}$ and non-AD patients [18]. This is especially the case in patients with dementia with Lewy bodies (DLB), due to concomitant AD pathology [19]. Nevertheless, diagnostic models based on $A \beta_{1-42}$ and $\mathrm{P}-\mathrm{tau}_{181 \mathrm{P}}$ are able to discriminate AD from a heterogeneous group of non-AD patients with an overall accuracy of more than $80 \%$ [3]. Biomarker-based diagnoses were found to be equally accurate as clinical diagnoses that were based on a complete clinical diagnostic work-up [3]. However, the added value of CSF biomarkers could lie within those cases in which the clinical diagnostic work-up is not able to discriminate between $\mathrm{AD}$ or non$\mathrm{AD}$ dementias. In the majority of clinically ambiguous cases, a correct diagnosis would have been established by means of the $\mathrm{AD}$ versus non-AD biomarker model [20].

Given the limited discriminatory power of the CSF biomarkers $\mathrm{A} \beta_{1-42}$, T-tau and $\mathrm{P}-\mathrm{tau}_{181 \mathrm{P}}$ for the differential diagnosis of dementia, new biomarkers are needed. One could think of other CSF biomarkers, like those that are reflective of the pathology of non-AD dementias. Combinations of CSF biomarkers with advanced MRI techniques or positron emission tomography (PET) with specific ligands might also help to improve the differential dementia diagnosis.

Besides improving the accuracy of $\mathrm{AD}$ diagnosis in clinical practice, the $\mathrm{AD}$ biomarkers will allow for an enrichment of $\mathrm{AD}$ cases in clinical trials to evaluate disease-modifying treatments. Once disease-modifying drugs will be available, biomarkers will be of help to establish a correct and early $\mathrm{AD}$ diagnosis, even in the preclinical stages of the disease. A correct diagnosis of $\mathrm{AD}$ is of importance as the potential disease-modifying treatments that are under study, will probably only be effective in $\mathrm{AD}$ and might have significant side effects. In case a disease-modifying treatment can be started in the preclinical phase of the disease, symptoms can subsequently be delayed or even prevented.

\section{References}

1. McKhann G, Drachman D, Folstein M, Katzman R, Price D, et al. (1984) Clinical diagnosis of Alzheimer's disease: report of the NINCDS-ADRDA Work Group under the auspices of Department of Health and Human Services Task Force on Alzheimer's Disease. Neurology 34: 939-944.

2. Engelborghs S, Sleegers K, Cras P, Brouwers N, Serneels S, et al. (2007) No association of CSF biomarkers with APOEepsilon4, plaque and tangle burden in definite Alzheimer's disease. Brain 130: 2320-2326.

3. Engelborghs S, De Vreese K, Van de Casteele T, Vanderstichele H, Van Everbroeck B, et al. (2008) Diagnostic performance of a CSF-biomarker panel in autopsy-confirmed dementia. Neurobiol Aging 29: 1143-1159.

4. Albert MS, Dekosky ST, Dickson D, Dubois B, Feldman HH, et al. (2011) The diagnosis of mild cognitive impairment due to Alzheimer's disease: recommendations from the National Institute on Aging-Alzheimer's Association workgroups on diagnostic guidelines for Alzheimer's disease. Alzheimers Dement 7: 270-279.

5. Jack CR Jr, Albert MS, Knopman DS, McKhann GM, Sperling RA, et al. (2011) Introduction to the recommendations from the National Institute on AgingAlzheimer's Association workgroups on diagnostic guidelines for Alzheimer's disease. Alzheimers Dement 7:257-262.
6. McKhann GM, Knopman DS, Chertkow H, Hyman BT, Jack CR Jr, et al.(2011) The diagnosis of dementia due to Alzheimer's disease: recommendations from the National Institute on Aging-Alzheimer's Association workgroups on diagnostic guidelines for Alzheimer's disease. Alzheimers Dement 7: 263-269.

7. Sperling RA, Aisen PS, Beckett LA, Bennett DA, Craft S, et al. (2011) Toward defining the preclinical stages of Alzheimer's disease: recommendations from the National Institute on Aging-Alzheimer's Association workgroups on diagnostic guidelines for Alzheimer's disease. Alzheimers Dement 7: 280-292.

8. Le Bastard N, Coart E, Vanderstichele H, Vanmechelen E, Martin JJ, et al. (2013) Comparison of two analytical platforms for the clinical qualification of Alzheimer's disease biomarkers in pathologically-confirmed dementia. J Alzheimers Dis 33: 117-131.

9. Mattsson N, Andreasson U, Persson S, Arai H, Batish SD, et al. (2011) The Alzheimer's Association external quality control program for cerebrospinal fluid biomarkers. Alzheimers Dement 7: 386-395

10. Vanderstichele H, Bibl M, Engelborghs S, Le Bastard N, Lewczuk P, et al (2012) Standardization of preanalytical aspects of cerebospinal fluid biomarke testing for Alzheimer's disease diagnosis: a consensus paper of the Alzheimer's Biomarkers Standaridzation Initiative. Alzheimers Dement 8: 65-73.

11. del Campo M, Mollenhauer B, Bertolotto A, Engelborghs $S$, Hampel H, et al. (2012) Recommendations to standardize preanalytical confounding factors in Alzheimer's and Parkinson's disease cerebrospinal fluid biomarkers: an update. Biomark Med 6: 419-430.

12. Engelborghs S, Le Bastard N (2012) The role of CSF biomarkers in the diagnostic work-up of mixed vascular-degenerative dementia. J Neurol Sci 322: 197-199.

13. Hansson O, Zetterberg H, Buchhave P, Londos E, Blennow K, et al. (2006) Association between CSF biomarkers and incipient Alzheimer's disease in patients with mild cognitive impairment: a follow-up study. Lancet Neurol 5 : 228-234.

14. Shaw LM, Vanderstichele H, Knapik-Czajka M, Figurski M, Coart E, et al. (2011) Qualification of the analytical and clinical performance of CSF biomarker analyses in ADNI. Acta Neuropathol 121: 597-609.

15. Visser PJ, Verhey F, Knol DL, Scheltens P, Wahlund LO, et al. (2009) Prevalence and prognostic value of CSF markers of Alzheimer's disease pathology in patients with subjective cognitive impairment or mild cognitive impairment in the DESCRIPA study: a prospective cohort study. Lancet Neurol 8: 619-627.

16. De Meyer G, Shapiro F, Vanderstichele H, Vanmechelen E, Engelborghs S et al. (2010) Diagnosis-independent Alzheimer disease biomarker signature in cognitively normal elderly people. Arch Neurol 67: 949-956.

17. Fagan AM, Roe CM, Xiong C, Mintun MA, Morris JC, et al. (2007) Cerebrospinal fluid tau/beta-amyloid(42) ratio as a prediction of cognitive decline in nondemented older adults. Arch Neurol 64: 343-349.

18. Blennow K, Hampel $H$, Weiner M, Zetterberg $H$ (2010) Cerebrospinal fluid and plasma biomarkers in Alzheimer disease. Nat Rev Neurol 6: 131-144.

19. Slaets S, Le Bastard N, Theuns J, Sleegers K, Verstraeten A, et al. (2013) Amyloid pathology influences AB1-42 cerebrospinal fluid levels in dementia with Lewy bodies. J Alzheimers Dis Jan 30. [Epub ahead of print].

20. Le Bastard N, Martin JJ, Vanmechelen E, Vanderstichele H, De Deyn PP, et al. (2010) Added diagnostic value of CSF biomarkers in differential dementia diagnosis. Neurobiol Aging 31: 1867-1876. 